This document is the Accepted Manuscript version of a Published Work that appeared in final form in J. Chem. Phys. 127, 174508 (2007), copyright (c) American Institute of Physics after peer review and technical editing by the publisher. To access the final edited and published work see

jcp.aip.org 


\title{
Nuclear quantum effects on the non-adiabatic decay mechanism of an excited hydrated electron
}

\author{
Daniel Borgis $^{\mathrm{a}, \mathrm{d}}$, Peter J. Rossky ${ }^{\mathrm{b}, \mathrm{d}}$ and László Turi ${ }^{\mathrm{c}, \mathrm{d}}$ \\ ${ }^{a}$ Département Physique et Modélisation, Université d'Evry-Val-d'Essone, Bd. François \\ Mitterand, 91025 Evry, France \\ ${ }^{b}$ Department of Chemistry and Biochemistry, Institute for Theoretical Chemistry, University \\ of Texas at Austin, Austin, TX 78712-1167 \\ ${ }^{\mathrm{c}}$ Department of Physical Chemistry, Eötvös Loránd University, Budapest 112, P.O.Box 32, \\ Hungary, H-1518
}

\begin{abstract}
We present a kinetic analysis of the non-adiabatic decay mechanism of an excited state hydrated electron to the ground state. The theoretical treatment is based on a quantized, gap dependent golden rule rate constant formula which describes the non-adiabatic transition rate between two quantum states. The rate formula is expressed in terms of quantum time correlation functions of the energy gap, and of the non-adiabatic coupling. These gap dependent quantities are evaluated from three different sets of mixed quantum-classical molecular dynamics simulations of a hydrated electron equilibrated a) in its ground state, b) in its first excited state, and c) on a hypothetical mixed potential energy surface which is the average of the ground and the first excited electronic states. The quantized, gap-dependent rate results are applied in a phenomenological kinetic equation which provides the survival probability function of the excited state electron. Although the lifetime of the equilibrated excited state electron is computed to be very short (well under $100 \mathrm{fs}$ ), the survival probability function for the non-equilibrium process in pump-probe experiments yields an effective
\end{abstract}

\footnotetext{
${ }^{\mathrm{d}}$ E-mail: daniel.borgis @ univ-evry.fr, fax: (33)-1-69-47-01-46 rossky@mail.utexas.edu, fax: (1)-512-471-3555 turi@chem.elte.hu, fax: (36)-1-372-2592.
} 
excited state lifetime of around $300 \mathrm{fs}$, a value consistent with the findings of several experimental groups and previous theoretical estimates. 


\section{Introduction}

The hydrated electron is a well known physical object that has been the subject of intensive experimental and theoretical research since its original identification in $1962 .{ }^{1}$ Although the cavity model of the hydrated electron is generally accepted, and structural, energetic and spectroscopic properties are understood in great detail, ${ }^{2}$ the hydrated electron system still retains some important uncertainties. One example is the origin of the shape of the experimentally measured optical absorption spectrum at high energies. ${ }^{3}$ Another, more controversial issue is the interpretation of pump-probe excitation experiments in terms of the contributions of ground and excited state solvent reorganization dynamics and excited state population decay. ${ }^{4,5,6,7,8,9,10} \mathrm{We}$ focus on this issue of excited state lifetime in the present work.

The non-adiabatic (NA) decay times for a p-state excited state electron back to the stype ground state, inferred from experiments, cover a remarkable range, presently from 50 to $\sim 1000$ fs. The three different basic scenarios behind these values assume a) a NA decay of the excited state within $\sim 200 \mathrm{fs}$, followed by a $\sim 1$ ps solvent relaxation around the ground state electron, put forth originally by Barbara et al., and later Assel et al.; ${ }^{4,5,8,9}$ b) an approximately 300 fs excited p-state solvent relaxation followed by a NA decay with a time constant around 1 ps proposed in more recent works by Barbara et al., ${ }^{6,7}$ and c) a much shorter p-state lifetime of $\sim 50$ fs inferred by Pshenichnikov et al. based on photon-echo experiments with very short (5 fs) pulses. ${ }^{10}$ A similar 50 fs time is obtained by Neumark and co-workers ${ }^{11}$ from results of measurements on anionic water clusters, extrapolated to infinite cluster size, in apparent agreement with photon-echo results. Nevertheless, recent experiments which probe the presence of the excited state electron more directly via selective scavengers ${ }^{12,13,14}$ appear to rule out such a short lifetime, consistent with classic work along these lines. ${ }^{15}$ These papers place the lifetime at $\sim 300-500$ fs. The different possible mechanistic models point to the 
ambiguity of the interpretation of the experimental signals. In this respect, evaluation of the experimental data in combination with theoretical studies can clarify the problem.

However, from the theoretical point of view, the results also cover a relatively wide range of timescales. Most importantly, the NA decay rates obtained from MD simulations seem to strongly depend on the interaction model, and the level of quantum simulation methodology employed. Staib and Borgis used a golden rule approach and obtained a NA decay time of $\sim 300 \mathrm{fs}$, with an equilibrated excited state trajectory, and a rigid, polarizable water model. ${ }^{16}$ Neria and Nitzan predicted $\sim 220$ fs with a similar approach but with a different flexible water model and incorporation of nuclear semi-classical (high-temperature) corrections. ${ }^{17,18}$ Schwartz and Rossky have performed direct non-adiabatic simulations of an electron excited from its ground state to one of the p-states, and they have monitored the subsequent relaxation, including solvent reorganization and surface hopping to the initial electronic state. ${ }^{19,20}$ They obtained an average p-state lifetime of $700 \mathrm{fs}$, and they inferred a lifetime of $450 \mathrm{fs}$ for an equilibrated p-state. The average lifetime of the excited state hydrated electron has also been computed with different non-adiabatic quantum/classical simulation algorithms by Larsen et al. predicting a range between 400 and $700 \mathrm{fs}^{21}$ More recently, very short population decay times of $50 \mathrm{fs}$ have been predicted by Zharikov and Fischer using a continuum hydrated electron model. ${ }^{22}$

In a previous paper, we have introduced a quantum time correlation formula for the NA decay rate between two adiabatic quantum states based on the Fermi golden rule. ${ }^{23}$ The lifetime of the equilibrium excited state electron has been predicted with the expression to be extremely short, $\sim 10$ fs. Although this result is in contrast with all previous simulations, we have also pointed out that the lifetime depends very strongly on the equilibrium energy gap, larger gaps resulting in significantly longer lifetimes. Based on our results, we concluded that the electronic relaxation after a photoexciting pulse is heavily influenced by solvent 
reorganization dynamics in the excited state. The aim of the present paper is to further examine the problem via the same Golden Rule route, and set up a realistic, gap dependent, kinetic rate equation from which one can estimate the lifetime of the excited state electron. In fact, we will show that the calculated mean lifetime of this non-equilibrium excited state is significantly longer than the hypothetical equilibrium lifetime, and, thus is consistent with the

measurements of Barbara et al., ${ }^{4,5,6,7,12}$ other recent measurements, ${ }^{13,14}$ and several previous MD simulation studies. ${ }^{16,18,19}$

The structure of the paper is as follows. In Sec. II we outline the theory, the full, quantum mechanical golden rule expression based on time correlation functions, its classical counterpart, and its quantized form in the harmonic quantization scheme. Sec III discusses the gap dependent decay rate in terms of a phenomenological first-order kinetic rate equation. The equation contains the gap-dependent rate constant and utilizes a linear response assumption. The details of the numerical simulations are also given in this section. We also provide an estimate of the lifetime of the excited state hydrated electron based on our considerations. Sec. IV concludes the paper.

\section{Background}

In our previous paper, we derived a quantum time correlation function expression for the non-adiabatic decay rate between two adiabatic states 1 and $2 .^{23}$

$$
\begin{aligned}
k_{1 \rightarrow 2} & =\frac{1}{\hbar^{2}} \int_{-\infty}^{\infty} d t\left[e^{i\left\langle\Omega_{12}(t)\right\rangle t} \times \exp \left\{-\int_{0}^{t} d \tau_{1} \int_{0}^{\tau_{1}} d \tau_{2}\left\langle\delta \Omega_{12}\left(\tau_{2}\right) \delta \Omega_{12}(0)\right\rangle\right\} \times\left\{\left\langle V_{12}(t) V_{21}(0)\right\rangle\right.\right. \\
& \left.\left.-\int_{0}^{t} \mathrm{~d} \tau_{1} \int_{0}^{t} \mathrm{~d} \tau_{2}\left\langle V_{12}\left(\tau_{1}\right) \Omega_{12}(0)\right\rangle \times\left\langle\Omega_{12}\left(\tau_{2}\right) V_{21}(0)\right\rangle\right\}\right]
\end{aligned}
$$

where $\delta$ stands for the fluctuations from the averages, $\hbar \Omega_{12}(t)$ describes the time dependent energy gap, and $V_{12}(t)$ is the time-dependent non-adiabatic coupling $\left(\left\langle V_{12}(t)\right\rangle=0\right) .{ }^{23}$ The formula involves the quantum correlation function of the coupling, the energy gap fluctuation, 
and their cross-terms. We verified that for the specific problem of the solvated electron, the cross terms are negligible, and the gap fluctuations fulfill the so-called slow modulation limit in which only the initial value determines the correlation function. ${ }^{23}$ Thus, the preceding equation reduces to

$$
\begin{aligned}
k_{1 \rightarrow 2} & =\frac{1}{\hbar^{2}} \int_{-\infty}^{\infty} d t \quad\left\langle V_{12}(t) V_{21}(0)\right\rangle \times \exp \left(i\left\langle\Omega_{12}\right\rangle t-\frac{1}{2}\left\langle\delta \Omega_{12}^{2}\right\rangle t^{2}\right) \\
& =\frac{1}{\hbar^{2}} \int_{-\infty}^{\infty} d t \quad C_{V}^{q}(t) \times \exp \left(i\left\langle\Omega_{12}\right\rangle t-\frac{1}{2} C_{\Omega}^{q}(0) t^{2}\right)
\end{aligned}
$$

where, in the second equality, we have introduced an obvious notation for the quantum coupling and frequency gap autocorrelation functions, $C_{V}^{q}(t)$ and $C_{\Omega}^{q}(t)$, respectively. Various formulae have been proposed to relate the quantum autocorrelation function of an arbitrary quantity $A, C^{q}(t)=\langle A(0) A(t)\rangle$, to its classical counterpart, $C(t) .{ }^{24,25,26,27,28,29,30} \mathrm{We}$ adopt here the so-called harmonic quantization scheme ${ }^{24,25}$ which is exact for harmonic systems. This scheme prescribes $\hat{C}^{q}(\omega)$ in the frequency domain as

$$
\hat{C}^{q}(\omega)=\frac{\beta \hbar \omega}{2 \tanh (\beta \hbar \omega / 2)} \times \frac{2}{1+e^{-\beta \hbar \omega}} \hat{C}(\omega)=\frac{\beta \hbar \omega}{1-e^{-\beta \hbar \omega}} \hat{C}(\omega)
$$

where $\hat{C}(\omega)$ is the Fourier transform of the classical correlation function $C(t), \hat{C}^{q}(\omega)$ is the quantized correlation function in the frequency domain, and $\beta=1 / k T$. In the first equality, the first term accounts for the renormalization of the individual mode amplitudes when going from the classical to the quantum regime, whereas the second one accounts for the detailed balance condition fulfilled by quantum correlation functions, $\hat{C}^{q}(-\omega)=e^{-\beta \hbar \omega} \hat{C}^{q}(\omega)$. This formula transforms in the time domain to

$$
C^{q}(t)=\int_{0}^{\infty} d \omega \frac{\hat{C}(\omega)}{\pi} \frac{\beta \hbar \omega}{2}(\operatorname{coth}(\beta \hbar \omega / 2) \cos (\omega t)-i \sin (\omega t)) .
$$


We think that this scheme is fully justified here for two reasons. First, for the energy gap, we have shown $^{23}$ that it does lead to the correct decoherence times for an excited pelectron when compared to the Gaussian packet propagation approach of Prezhdo and Rossky, ${ }^{31}$ and Turi and Rossky, ${ }^{32}$ both for water and methanol. Second, the coupling fluctuations turn out to be strongly dominated by the librational and vibrational solvent modes, ${ }^{23}$ and a harmonic bath description (for which the formula is exact) is quite sensible here. The rate formula, eq. (2), can be easily transformed from the time to the frequency domain to yield ${ }^{23}$

$$
k_{1 \rightarrow 2}=\int_{-\infty}^{+\infty} d \omega \hat{k}^{q}(\omega)
$$

where $\hat{k}^{q}(\omega)$ gives the contribution of the frequency $\omega$ to the total rate. The frequency dependent rate contribution then reads as

$$
\hat{k}^{q}(\omega)=\frac{1}{\hbar^{2}} \hat{C}_{V}^{q}(\omega) \hat{W}(\omega)
$$

$\hat{W}(\omega)$ is a "window function" defined by

$$
\hat{W}(\omega)=\left(2 \pi\left\langle\delta \Omega_{12}^{2}\right\rangle_{q}\right)^{-1 / 2} e^{-\frac{\left(\omega-\left\langle\Omega_{12}\right\rangle\right)^{2}}{2\left\langle\delta \Omega_{12}^{2}\right\rangle_{q}}}
$$

which favors the solvent modes with frequencies around the mean energy gap (a LandauTeller picture $\left.{ }^{23,33,34}\right)$. In eq (6), $\hat{C}_{V}^{q}(\omega)$ stands for the quantum coupling correlation function in the frequency domain.

$$
C_{V}^{q}(t)=\frac{1}{2 \pi} \int_{-\infty}^{+\infty} d \omega e^{i \omega t} \hat{C}_{V}^{q}(\omega)
$$

We note that eqs (6)-(7) correct a slight typographical error in Reference 23 (eqs (34), (35)) and define correctly $\hat{W}(\omega)$ as a normalized function tending to the Dirac function in the zero- 
fluctuation Landau-Teller limit. The quantum frequency gap variance $\left\langle\delta \Omega_{12}^{2}\right\rangle_{q}=C_{\Omega}^{q}(0)$ is related to its classical counterpart through eq. (4), i.e,

$$
\left\langle\delta \Omega_{12}^{2}\right\rangle_{q}=\left\langle\delta \Omega_{12}^{2}\right\rangle \int_{0}^{\infty} d \omega \frac{\tilde{C}_{\Omega}(\omega)}{\pi} \frac{\beta \hbar \omega}{2} \operatorname{coth}\left(\frac{\beta \hbar \omega}{2}\right)
$$

Here $\tilde{C}_{\Omega}(\omega)$ is the Fourier transform of the normalized, classical energy-gap correlation function. Using the harmonic quantization scheme, the following final expression is easily obtained for the frequency-dependent rate: $:^{23}$

$$
\hat{k}^{q}(\omega)=\frac{1}{\sqrt{2 \pi} \hbar^{2}}\left\langle V_{12}^{2}\right\rangle\left\langle\delta \Omega_{12}^{2}\right\rangle_{q}^{-1 / 2} \frac{\beta \hbar \omega}{1-e^{-\beta \hbar \omega}} \tilde{C}_{V}(\omega) e^{-\frac{\left(\omega-\left\langle\Omega_{12}\right\rangle\right)^{2}}{2\left\langle\delta \Omega_{12}^{2}\right\rangle_{q}}}
$$

$\tilde{C}_{V}(\omega)$ is defined here as the Fourier transform of the normalized, classical coupling correlation function. That formula can be compared to the "classical" version, in which the nuclear modes dynamics are considered as purely classical

$$
\hat{k}(\omega)=\frac{1}{\sqrt{2 \pi} \hbar^{2}}\left\langle V_{12}^{2}\right\rangle\left\langle\delta \Omega_{12}^{2}\right\rangle^{-1 / 2} \tilde{C}_{V}(\omega) e^{-\frac{\left(\omega-\left\langle\Omega_{12}\right\rangle\right)^{2}}{2\left\langle\delta \Omega_{12}^{2}\right\rangle}}
$$

\section{Gap dependent decay rate}

In our previous work, we applied these formulae to the decay rate from an equilibrated p-electron to the ground state s-electron. ${ }^{23}$ The extremely short times found for both the classical and quantum cases (well below $100 \mathrm{fs)} \mathrm{pointed} \mathrm{to} \mathrm{the} \mathrm{fact} \mathrm{that} \mathrm{the} \mathrm{equilibrated} \mathrm{p-}$ electron might be a hypothetical state that is, in fact, never realized. During the energy gap relaxation process, nonadiabatic transitions can occur at higher energy gaps, before the minimal, equilibrium gap is reached.

To model this, we proposed to extend the above formula to arbitrary energy gaps by varying the quantity $\left\langle\Omega_{12}\right\rangle$ in the rate formula (9). This quantity enters in two major ways: 
first in the exponent of the window function, and second, implicitly in the averaged squared coupling element of the prefactor. The other relevant quantities, the normalized coupling correlation function and energy gap correlation function in eqs (9)-(11), are assumed to have much weaker dependence; this assumption will be confirmed below. Treating the implicit dependence of coupling on gap, and neglecting dependence on quantum fluctuations in structure, $\left\langle V_{12}^{2}\right\rangle$ can be written as

$$
\left\langle V_{12}^{2}\right\rangle\left(\left\langle\Omega_{12}\right\rangle\right)=\int_{0}^{\infty} d \Omega V_{12}^{2}(\Omega) P(\Omega) \approx \int_{0}^{\infty} d \Omega V_{12}^{2}(\Omega)\left(2 \pi\left\langle\delta \Omega_{12}^{2}\right\rangle_{q}\right)^{-1 / 2} e^{-\frac{\left(\Omega-\left\langle\Omega_{12}\right\rangle\right)^{2}}{2\left\langle\delta \Omega_{12}^{2}\right\rangle_{q}}}
$$

where $V_{12}^{2}(\Omega)$ is the averaged value of the squared coupling sampled for a given realization of the instantaneous frequency gap, $\Omega$, whereas $P(\Omega)$ is the corresponding quantum frequency gap probability distribution function. In the second equality, this probability distribution has been assumed to be Gaussian; in this limit, it becomes identical to the Window function of eq. (7).

To determine $\left\langle V_{12}^{2}\right\rangle\left(\left\langle\Omega_{12}\right\rangle\right)$, we have performed three different simulations of a hydrated electron: equilibrated in its ground s-state, in the first p-excited state, and in a state where the solvent responds to the average of the lowest two Born-Oppenheimer electronic states (mid-state), corresponding to energy $\frac{1}{2}\left(E_{s}+E_{p}\right)$. The basics of the method can be found in Ref. 35. The details of the actual simulations are similar to our previous simulations in Ref. 36. The solvent bath consists of 1600 water molecules in a cubic simulation cell. The molecular interactions are described by a three-site classical model potential with added internal flexibility. The electron is treated quantum mechanically in a plane wave basis and is represented on $16^{3}$ grid points equidistantly distributed in a box, with the edge length equal to half of the length of the simulation cell. The interaction between the quantum particle and the 
classical molecules is given by a model pseudopotential. ${ }^{36}$ The nuclear configurations are adiabatically propagated on the potential surfaces using the sum of classical and HellmannFeynman forces. The simulation time step is 1 fs. For the ground state and the mid-state trajectories, we have used a smooth spherical cut-off with a tapering function for the solventsolvent and the solvent-electron interactions. Due to observed instability of the excited state trajectory, ${ }^{23}$ the long-range part of the interactions and the forces are calculated for this trajectory using the Ewald summation technique including solvent-solvent and the solventelectron interactions explicitly, similar to the work of Rossky and co-workers. ${ }^{37}$

The probability distributions of the energy gap are shown in Figure 1. The Gaussian character assumed in the preceding equation is seen to be well fulfilled in the three cases, although less well for the excited state. The measured average values are $1.84,1.01$, and 0.43 $\mathrm{eV}$ in the ground, mid, and excited state, respectively. The standard deviation is quite similar in the three cases. However, it is not constant and, in fact, non-monotonic with values of 0.17 , $0.20,0.16 \mathrm{eV}$, respectively, the variation in boundary conditions may play a role in this.

The gap dependent coupling $V_{12}^{2}(\Omega)$ sampled independently from the three simulations is displayed in figure 2. It can be seen that all data nicely lie on a single common master curve, which can be fitted by a one parameter function of the form $V_{12}^{2}(\Omega) / \hbar^{2}=a /(\hbar \Omega)^{2}$ ( $a=4.4 \times 10^{-4}$, and $\hbar \Omega$ is in $\mathrm{eV}$ ). We note that this dependence is to be expected based on the quantum-classical expression of the non-adiabatic coupling matrix elements. ${ }^{38,39}$

The evaluation of the gap dependent rate relies on the hypothesis that the normalized energy gap and coupling correlation functions are relatively independent of the reference state. This is confirmed, as seen by comparison of the ground state and the mid-state in figures 3 and 4. The energy gap correlation functions in Fig. 3 are very close to each other, and they are reminiscent of the equilibrium and nonequilibrium relaxation curves computed by Schwartz and Rossky (although with a different pseudopotential). ${ }^{19}$ The gap curves present 
a typical multi-exponential character, which could be well fitted by a function of the form, $\tilde{C}_{\Omega}(t) \approx 0.232 e^{-t^{2} / 178}+0.342 e^{-t / 60}+0.426 e^{-t / 700}$. As we observed previously for excited state simulation, ${ }^{23}$ the coupling fluctuations appear to be strongly coupled to the librational and vibrational solvent modes (see also Fig. 4), and there is little contribution from translational and rotational motions. The ground and mid-state situations are very similar, and the spectral density of the coupling, defined conventionally as $\tilde{C}_{V}(\omega) / \pi$, presents three peaks, around $800 \mathrm{~cm}^{-1}$ (water librations), $1700 \mathrm{~cm}^{-1}$ (bending), and $3500 \mathrm{~cm}^{-1}$ (symmetric and antisymmetric stretch). Those peaks, particularly the libration and stretch regions, are somewhat distorted between the ground and excited state. This distortion reflects the change in coupling strength between the electron and modes of different symmetry with a change in electronic shape (s vs. p). ${ }^{40}$ In Fig. 4, we also compare the quantized and classical spectral densities, and, in this vibrational frequency range, the quantum effects are indeed very important, and are dominated by the mode amplitude renormalization factor, $\beta \hbar \omega / 2 \tanh (\beta \hbar \omega / 2)$.

In Fig. 5, we display the energy-gap dependent decay time, $\tau_{1 \rightarrow 2}\left(\left\langle\Omega_{12}\right\rangle\right)=1 / k_{1 \rightarrow 2}\left(\left\langle\Omega_{12}\right\rangle\right)$, obtained using the previous prescriptions for the energy-gap dependent coupling, and the mid-state dynamics for defining the time-dependent correlation functions. The classical and quantum results are quite similar to the ones presented in our previous reference, ${ }^{23}$ although, there, everything was adjusted to the excited state properties, and the coupling dependence was not correctly described beyond the energy gap range explored in the simulations, i.e. above $\sim 0,8 \mathrm{eV}$. It can be concluded again that the nuclear dynamics quantization leads to a huge (one-order of magnitude) decrease of the computed, classical non-adiabatic transition time.

The energy-gap dependence, being known in the whole energy range between ground and excited state situations, can be used to construct a phenomenological kinetic equation for 
the survival probability of a p-electron, excited at time zero from its equilibrated ground state. For this, instead of calculating the response directly from non-equilibrium trajectories, we rely on a linear response approximation relating the non-equilibrium energy gap relaxation response following a transition from state 2 to state 1 at $t=0$ to an equilibrium ground state energy-gap correlation function, i.e

$$
S(t)=\frac{\bar{\Omega}(t)-\left\langle\Omega_{12}\right\rangle_{1}}{\left\langle\Omega_{12}\right\rangle_{2}-\left\langle\Omega_{12}\right\rangle_{1}}=\frac{\left\langle\delta \Omega_{12}(0) \delta \Omega_{12}(t)\right\rangle_{2}}{\left\langle\delta \Omega_{12}^{2}\right\rangle_{2}}=\tilde{C}_{\Omega}(t),
$$

yielding the expression of the average gap frequency $\bar{\Omega}(t)$ in a non-equilibrium relaxation process as a function of $\tilde{C}_{\Omega}(t)$

$$
\bar{\Omega}(t)=\left\langle\Omega_{12}\right\rangle_{1}+\left(\left\langle\Omega_{12}\right\rangle_{2}-\left\langle\Omega_{12}\right\rangle_{1}\right) \tilde{C}_{\Omega}(t)
$$

The validity of this linear response relationship for the hydrated electron problem was illustrated by Schwartz and Rossky. ${ }^{19}$ We can then make use of the same phenomenological equation that they used to describe the time evolution of the survival probability of the excited p-state electron

$$
\frac{d P}{d t}=-k_{1 \rightarrow 2}(\bar{\Omega}(t)) P(t) .
$$

Since $\bar{\Omega}(t)$ and $k_{1 \rightarrow 2}(\bar{\Omega}(t))$ are known, this equation can be easily integrated numerically to yield the curves displayed in Fig 6 for each of the classical and quantized cases. The classical curve obtained is very similar to the one obtained by Rossky and Schwartz by monitoring transition times in a set of independent non-adiabatic, non-equilibrium trajectories. ${ }^{19} \mathrm{We}$ observe a similar inflexion point around $700 \mathrm{fs}$, and the inferred decay time, defined by the integral of $P(t)$, is $\sim 900$ fs. On the other hand, in the quantum case, the non-adiabatic transitions occur much earlier, as expected from the times plotted in Fig. 5. The inflexion point occurs around $250 \mathrm{fs}$ and the apparent lifetime is $\sim 330 \mathrm{fs}$. 


\section{Discussion and conclusions}

We have extended our earlier development of a quantized time-dependent formulation of the Golden Rule ${ }^{23}$ with an explicit electronic energy gap dependence for the treatment of non-equilibrium electronic excited state decay. This rate expression is used in concert with a linear response description of solvation dynamics to obtain a self-contained kinetic expression for the excited state survival probability. A convenient implementation is described which depends on several approximations. Among these are linear response for the fluctuations in energy and non-adiabatic coupling and a separation of time scales between fluctuations in the energy gap and those in the non-adiabatic coupling. The methods are applied to the problem of the excited state relaxation of the hydrated electron following photoexcitation from the ground state. We have verified that the approximations made in the formal development are valid for this application and applied the methodology using three separate equilibrium adiabatic simulations to span the range of energy gaps. The implicit dependence of the nonadiabatic coupling on the energy gap is included in the calculation as well.

The resulting survival probabilities show clearly that the very short lifetime of the hypothetical equilibrated excited state is not closely connected to the observed survival time in the pump-probe experiment, resolving that issue in our earlier work. ${ }^{23}$ Specifically, the non-equilibrium results obtained here using a fully classical solvent bath correspond well to those obtained from the statistics of full mixed quantum-classical non-adiabatic dynamics simulations published earlier. ${ }^{19,21}$ The apparent lifetime in this classical case is a bit less than 1 ps. The results obtained here with a quantum bath provide a decay profile of similar shape, but with a shorter apparent lifetime of $\sim 330 \mathrm{fs}$. This value is quite consistent with the values inferred from both pump-probe experiments ${ }^{4,5,6,7}$ and independent excited state electron scavenging kinetics ${ }^{12,13,14}$ which put the lifetime in the $\sim 300-500$ fs range. As noted in the 
Introduction, a value for the lifetime of $\sim 50 \mathrm{fs}$, inferred from photon-echo experiments, ${ }^{10}$ and also inferred from extrapolation of excited state dynamics of anionic water clusters, ${ }^{11}$ are not consistent with the interpretations given to these several other experiments. We conclude here that the present atomistic theoretical results clearly support the assignment of the longer lifetime to the electron excited state, in accord with reports of pump-probe experiments and scavenging experiments, and also in accord with earlier fully atomistic simulations of hydrated electron non-adiabatic dynamics.

\section{Acknowledgments}

L.T. gratefully acknowledges the tenure of the Bolyai Research Fellowship, and the Öveges Research Fellowship. The work was supported by grants to L. T. from the National Research Fund of Hungary (OTKA) under Contracts No. T049715 and IN69707. P. J. R. is grateful to the National Science Foundation (CHE-0615173) and the R. A. Welch Foundation (F-0019) for support of this work. 


\section{Figure Captions}

Figure 1. The s-p energy gap ( $E=\hbar \Omega$ ) probability distribution for an electron equilibrated in its ground state (right), first excited state (left), and in a hypothetical state, which is the average of the ground and excited states (middle). Symbols show the simulation results, solid lines correspond to the Gaussian approximation.

Figure 2. Frequency-dependent squared non-adiabatic coupling collected for the three different simulations in the ground state, mid-state, and excited state (squares, triangles, and circles, respectively). The solid line corresponds to the fit $V_{12}^{2}(\Omega) / \hbar^{2}=4.4 \times 10^{-4} /(\hbar \Omega)^{2}$.

Figure 3. Normalized energy gap correlation function $\left(C_{\Omega}(t) / C_{\Omega}(0)\right)$ for the ground and mid-state (dashed line and solid line, respectively).

Figure 4. Spectral density of the normalized coupling correlation function, $\tilde{C}_{V}(\omega) / \pi$. Top: Classical spectra compared for two states: mid-state, (solid line), ground state (dashed line). Bottom: Quantized vs. classical mid-state spectrum: quantum (solid line), classical (dashed line).

Figure 5. Gap-dependent decay time $\left(\tau_{1 \rightarrow 2}\left(\left\langle\Omega_{12}\right\rangle\right)=1 / k_{1 \rightarrow 2}\left(\left\langle\Omega_{12}\right\rangle\right)\right)$ for the excited p-state electron back to the ground state, using the correlation functions of the mid-state trajectory. The results for the classical case (dashed line) and the harmonic quantization scheme (solid line) are shown. 
Figure 6. Survival probability as a function of time for the classical (dashed line) and quantum cases (solid line), computed from the phenomenological kinetic equation (eq. (14)). 
Figure 1. Borgis, Rossky, and Túri

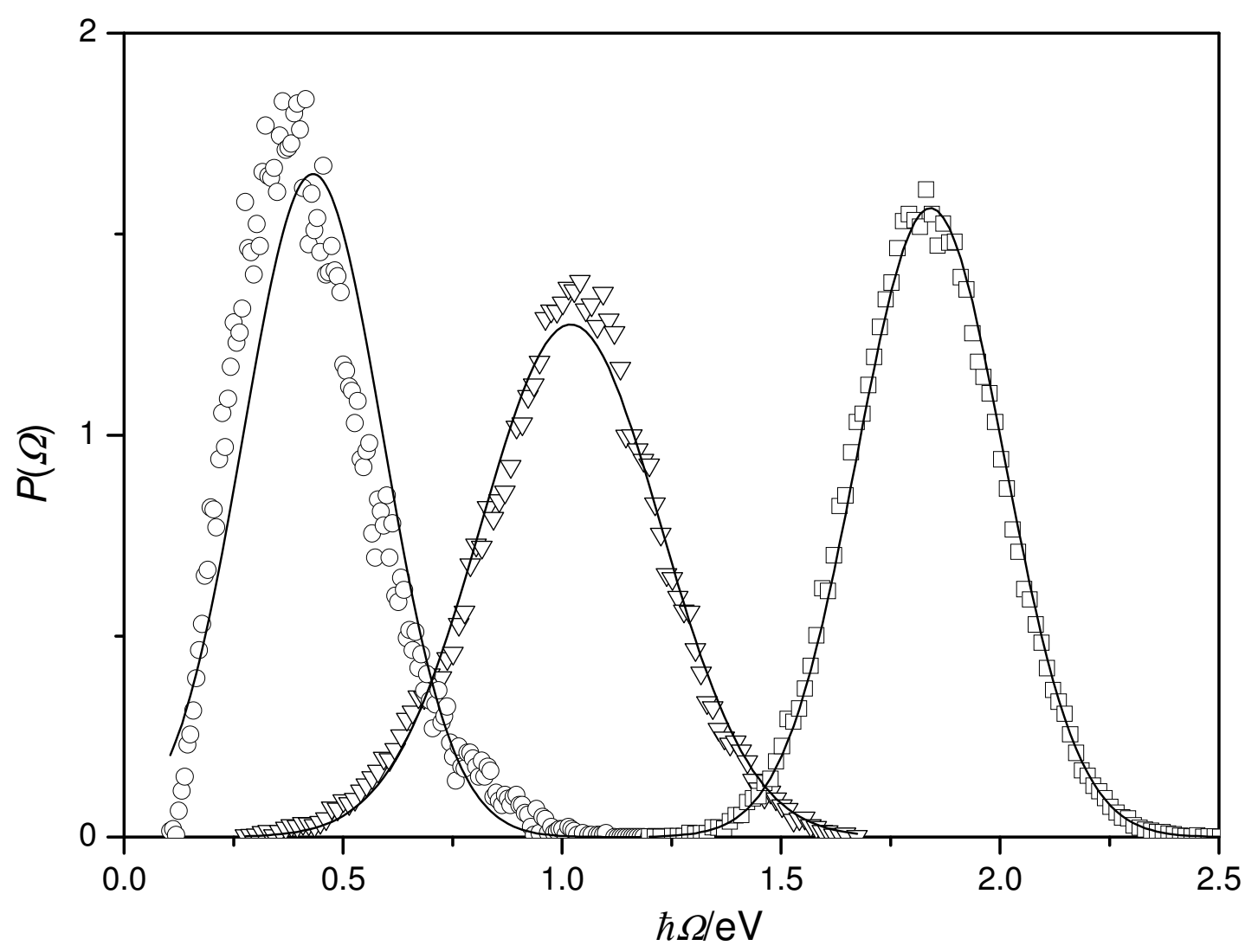


Figure 2. Borgis, Rossky, and Túri

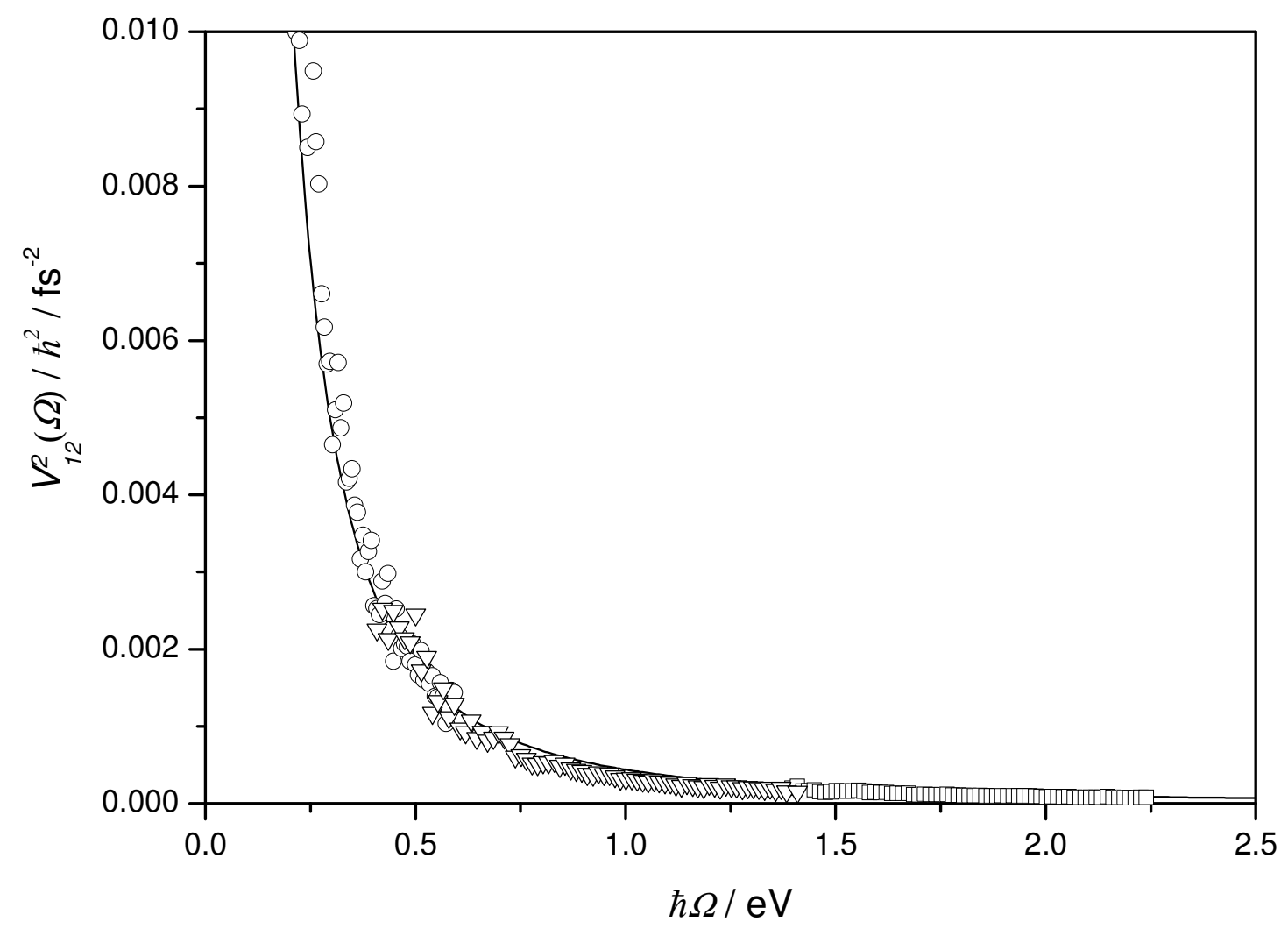


Figure 3. Borgis, Rossky, and Túri

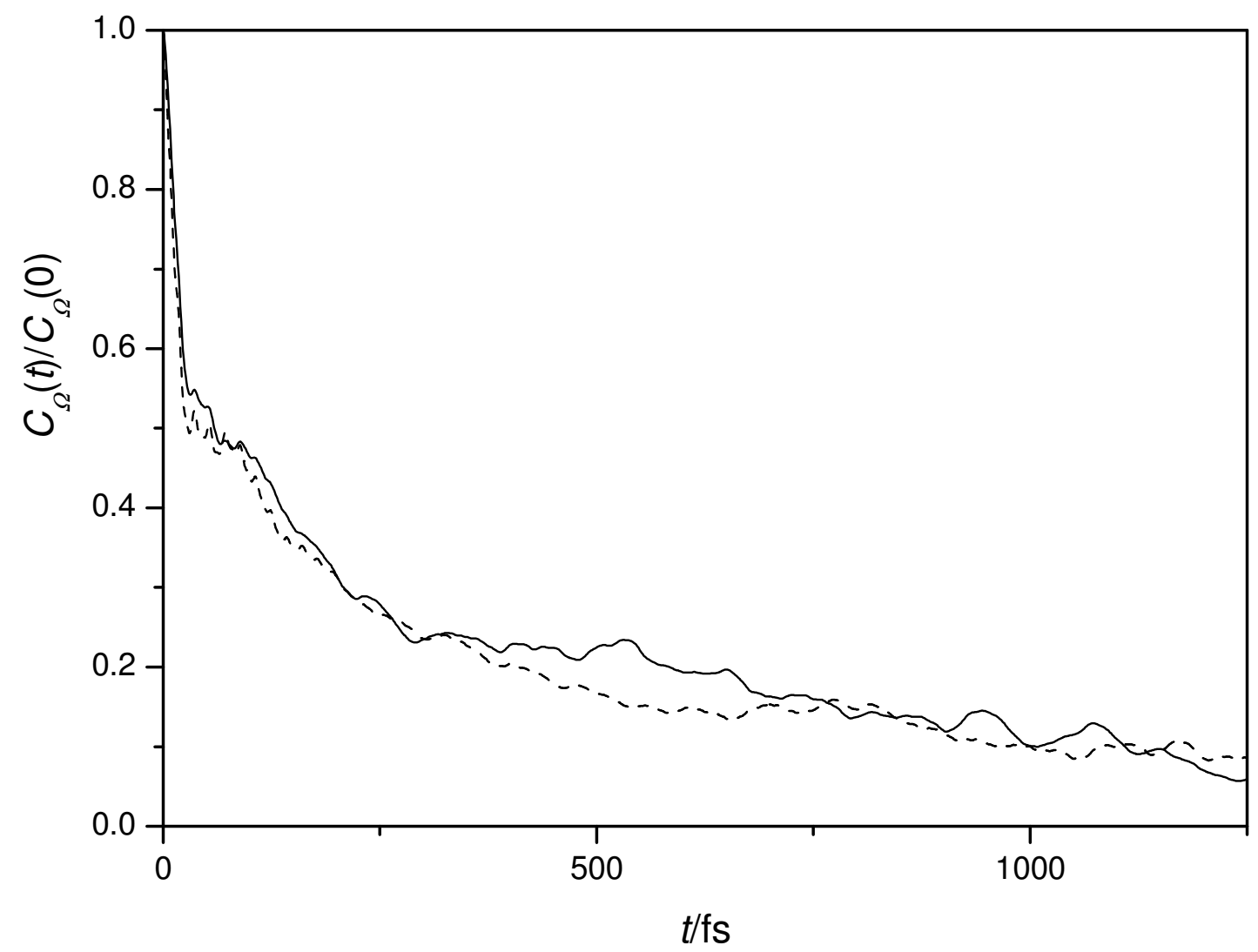


Figure 4. Borgis, Rossky, and Túri
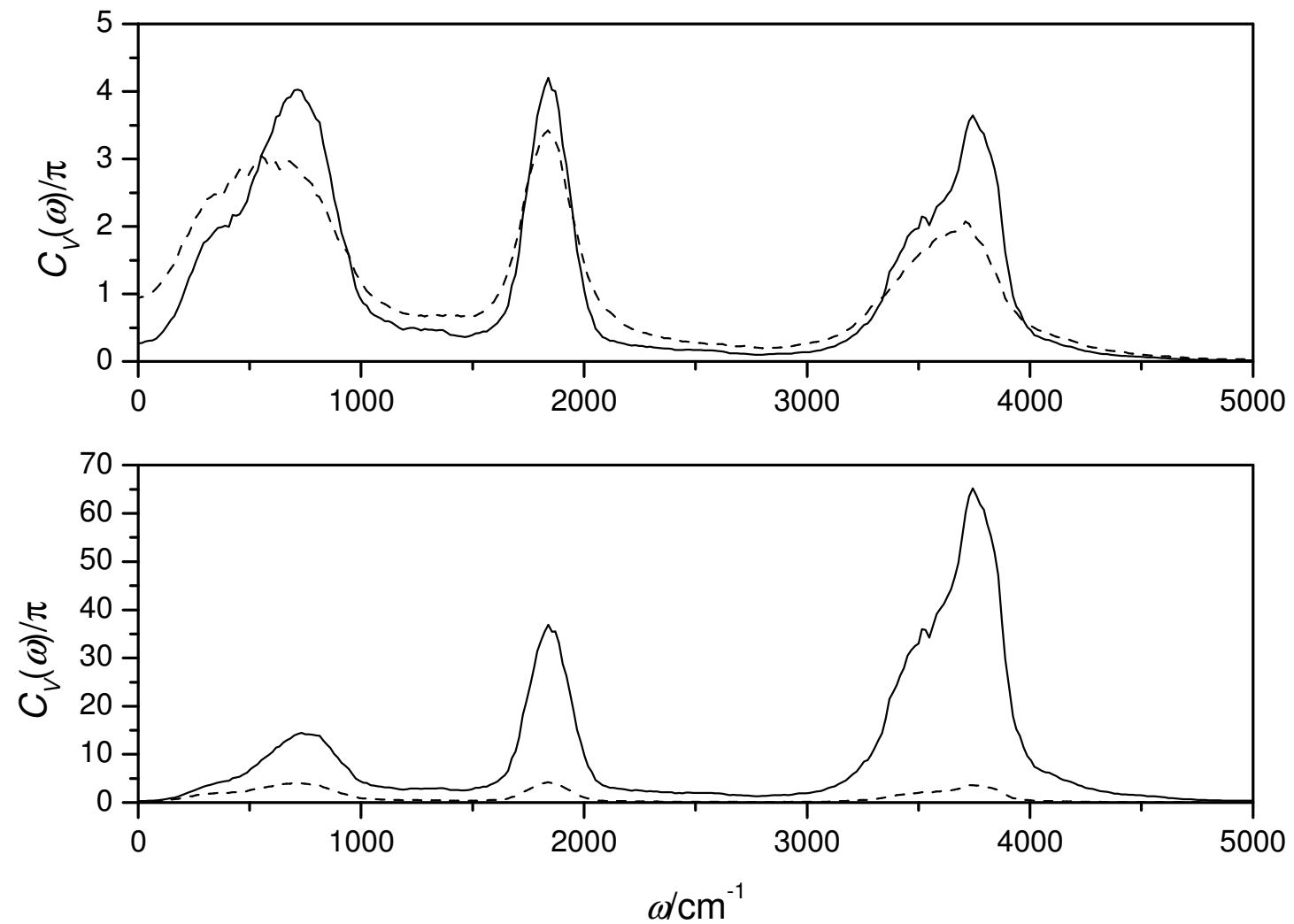
Figure 5. Borgis, Rossky, and Túri

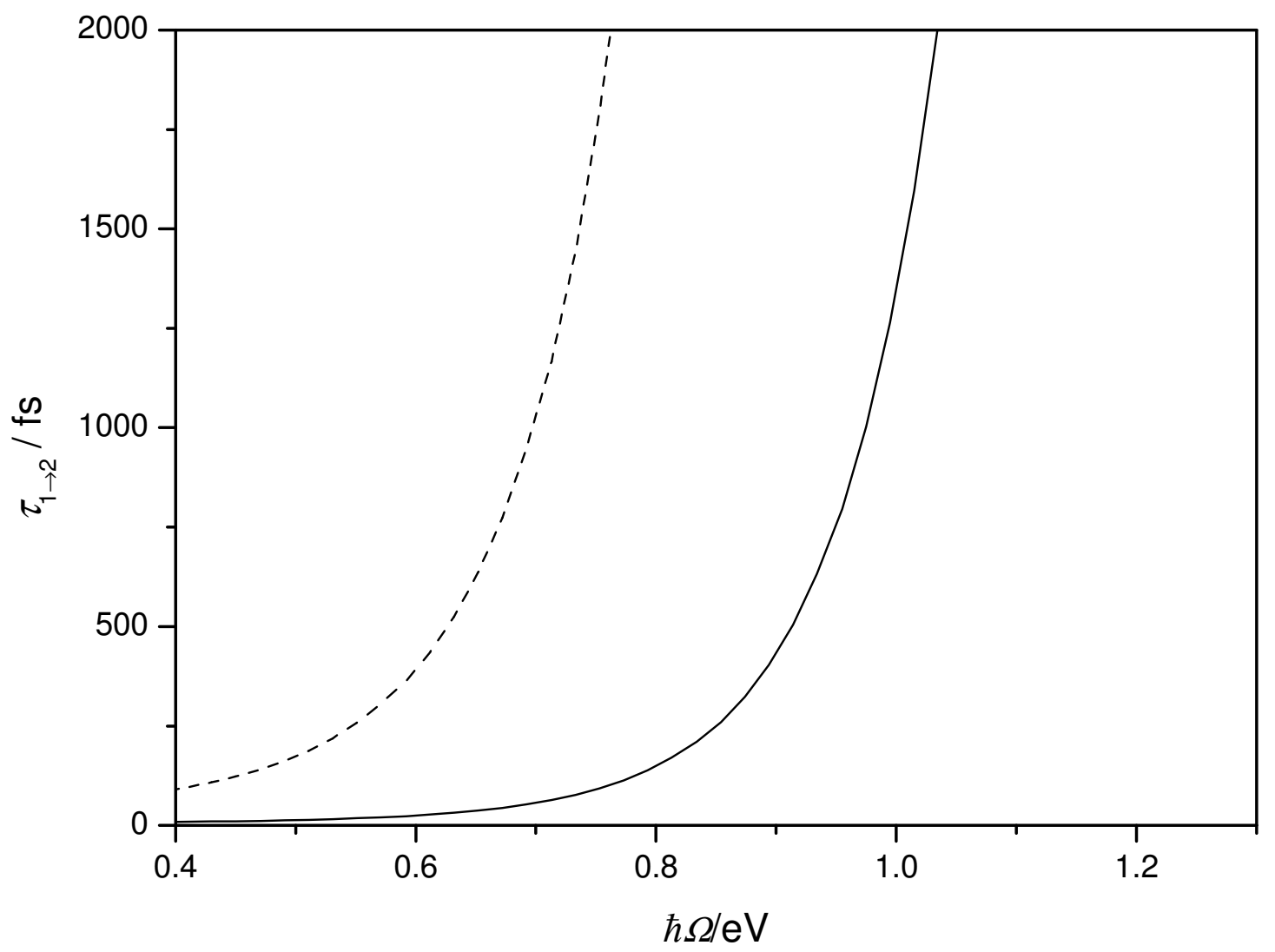


Figure 6. Borgis, Rossky, and Túri

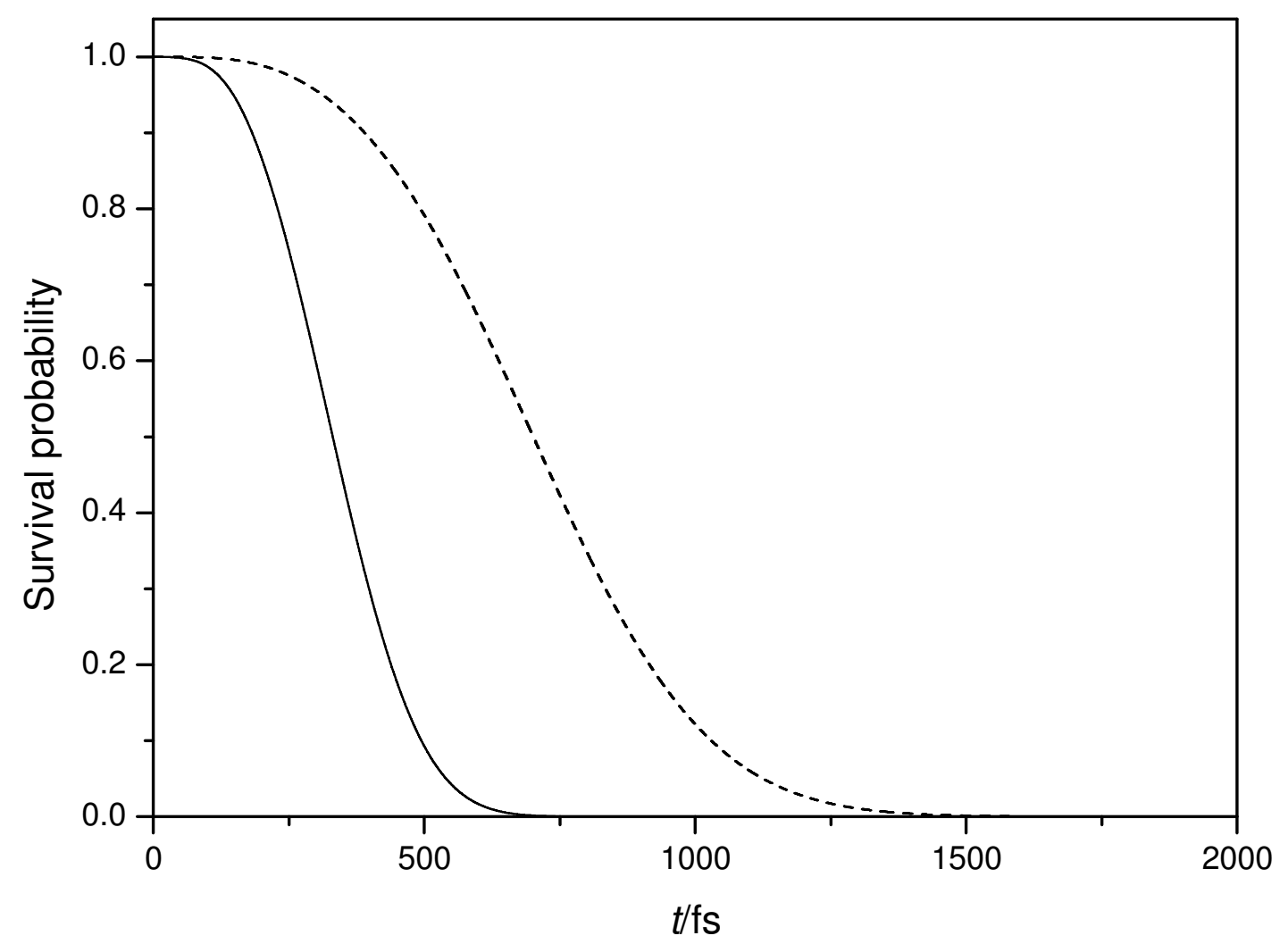




\section{References}

${ }^{1}$ E. J. Hart and J. W. Boag, J. Am. Chem. Soc. 84, 4090 (1962).

${ }^{2}$ P. J. Rossky and J. Schnitker, J. Phys. Chem. 92, 4277 (1988).

${ }^{3}$ F.-Y. You and G. R. Freeman, J. Phys. Chem. 81, 909 (1977).

${ }^{4}$ J. C. Alfano, P. K. Walhout, Y. Kimura, and P. F. Barbara, J. Chem. Phys. 98, 5996 (1993).

${ }^{5}$ P. J. Reid, C. Silva, P. K. Walhout, and P. F. Barbara, J. Phys. Chem. 98, 3450 (1994).

${ }^{6}$ K. Yokoyama, C. Silva, D. H. Son, P. K. Walhout, and P. F. Barbara, J. Phys. Chem. A 102, 6957 (1998).

${ }^{7}$ C. Silva, P. K. Walhout, K. Yokoyama, and P. F. Barbara, Phys. Rev. Lett. 80, 1086 (1998).

${ }^{8}$ M. Assel, R. Laenen, and A. Laubereau, J. Phys. Chem. A 102, 2256 (1998).

${ }^{9}$ M. Assel, R. Laenen, and A. Laubereau, Chem. Phys. Lett. 317, 13 (2000).

${ }^{10}$ M. S. Pshenichnikov, A. Baltuska, and D. W. Wiersma, Chem. Phys. Lett. 389, 171 (2004).

${ }^{11}$ J. R. R. Verlet, A. E. Bragg, A. Kammrath, O. Cheshnovsky, and D. M. Neumark, Science 307, 93 (2005).

${ }^{12}$ T. W. Kee, D. H. Son, P. Kambhampati, and P. F. Barbara, J. Phys. Chem. A 105, 8434 (2001).

${ }^{13}$ C.-R. Wang, A. Hu, and Q.-B. Lu, J. Chem. Phys. 124, 241102 (2006).

${ }^{14}$ C.-R. Wang, and Q.-B. Lu, Angew. Chem. Intl. Ed., accepted for publication.

${ }^{15}$ A. C. Chernovitz, and C. D. Jonah, J. Phys. Chem. 92, 5946 (1988).

${ }^{16}$ A. Staib and D. Borgis, J. Chem. Phys. 103, 2642 (1995).

${ }^{17}$ E. Neria, A. Nitzan, R. N. Barnett, and U. Landman, Phys. Rev. Lett. 67, 1011 (1991).

${ }^{18}$ E. Neria and A. Nitzan, J. Chem. Phys. 99, 1109 (1993).

${ }^{19}$ B. J. Schwartz and P. J. Rossky, J. Chem. Phys. 101, 6902 (1994).

${ }^{20}$ B. J. Schwartz and P. J. Rossky, J. Chem. Phys. 101, 6917 (1994).

${ }^{21}$ R. E. Larsen, M. J. Bedard-Hearn, and B. J. Schwartz, J. Phys. Chem. B 110, 20055 (2006). 
${ }^{22}$ A. A. Zharikov and S. F. Fischer, J. Chem. Phys. 124, Art. No. 054506 (2006).

${ }^{23}$ D. Borgis, P. J. Rossky, and L. Turi, J. Chem. Phys. 125, 064501 (2006).

${ }^{24}$ P. H. Berens, S. R. White, and K. R. Wilson, J. Chem. Phys. 75, 515 (1981).

${ }^{25}$ J. S. Bader and B. J. Berne, J. Chem. Phys. 100, 8359 (1994).

${ }^{26}$ B. J. Berne and G. D. Harp, Adv. Chem. Phys. 17, 63 (1970).

${ }^{27}$ D. W. Oxtoby, Adv. Chem. Phys. 47, 487 (1981).

${ }^{28}$ P. Schofield, Phys. Rev. Lett. 4, 239 (1960).

${ }^{29}$ P. A. Egelstaff, Adv. Phys. 11, 203 (1962).

${ }^{30}$ H. Kim and P. J. Rossky J. Phys. Chem. B 106, 8240 (2002).

${ }^{31}$ O. V. Prezhdo and P. J. Rossky, J. Chem. Phys. 107, 5863 (1997).

${ }^{32}$ L. Turi and P. J. Rossky, J. Chem. Phys. 120, 3688 (2004).

${ }^{33}$ R. M. Whitnell, K. R. Wilson, and J. T. Hynes, J. Chem. Phys. 96, 5354 (1992).

${ }^{34}$ K. F. Everitt, J. L. Skinner, and B. M. Ladanyi, J. Chem. Phys. 116, 179 (2002).

${ }^{35}$ F. A. Webster, P. J. Rossky, and R. A. Friesner, Comput. Phys. Commun. 63, 494 (1991).

${ }^{36}$ L. Turi and D. Borgis, J. Chem. Phys. 114, 6186 (2002).

${ }^{37}$ C.-Y. Yang, K. F. Wong, M. S. Skaf, and P. J. Rossky, J. Chem. Phys. 114, 3598 (2001).

${ }^{38}$ N. F. Mott, Proc. Cambridge Philos. Soc. 27, 553 (1931).

${ }^{39}$ E. Neria, A. Nitzan, R. N. Barnett, U. Landman, Phys. Rev. Lett. 67, 1011 (1991).

${ }^{40}$ O. V. Prezhdo, and P. J. Rossky, J. Phys. Chem. 100, 17094 (1996). 